\title{
Anonymous Quantum Nonlocality
}

\author{
Yeong-Cherng Liang, ${ }^{1}$ Florian John Curchod, ${ }^{2,3}$ Joseph Bowles, ${ }^{4}$ and Nicolas Gisin ${ }^{3}$ \\ ${ }^{1}$ Institute for Theoretical Physics, ETH Zurich, 8093 Zurich, Switzerland. \\ ${ }^{2}$ ICFO-Institut de Ciències Fotòniques, 08860 Castelldefels (Barcelona), Spain. \\ ${ }^{3}$ Group of Applied Physics, University of Geneva, CH-1211 Geneva 4, Switzerland. \\ ${ }^{4}$ Department of Theoretical Physics, University of Geneva, 1211 Geneva, Switzerland.
}

(Dated: July 23, 2018)

\begin{abstract}
We show that for all $n \geq 3$, an example of an $n$-partite quantum correlation that is not genuinely multipartite nonlocal but rather exhibiting anonymous nonlocality, that is, nonlocal but biseparable with respect to all bipartitions, can be obtained by locally measuring the $n$-partite GreenbergerHorne-Zeilinger (GHZ) state. This anonymity is a manifestation of the impossibility to attribute unambiguously the underlying multipartite nonlocality to any definite subset(s) of the parties, even though the correlation can indeed be produced by nonlocal collaboration involving only such subsets. An explicit biseparable decomposition of these correlations is provided for any partitioning of the $n$ parties into two groups. Two possible applications of these anonymous GHZ correlations in the device-independent setting are discussed: multipartite secret sharing between any two groups of parties and bipartite quantum key distribution that is robust against nearly arbitrary leakage of information.
\end{abstract}

Quantum correlations that violate a Bell inequality [1], a constraint first derived in the studies of local-hiddenvariable-theories, were initially perceived only as a counterintuitive feature with no classical analog. With the discovery of quantum information science, these intriguing correlations have taken the new role as a resource. For instance, in nonlocal games [2], the presence of Bellinequality-violating (hereafter referred as nonlocal) correlation signifies the usage of strategies that cannot be achieved using only shared randomness. They are also an indispensable resource in quantum information and communication tasks such as the reduction of communication complexity [3], the distribution of secret keys using untrusted devices [4, 5], as well as the certification and expansion of randomness [6] etc. (see 7] for a review).

Thus far, prior studies of quantum nonlocality have focussed predominantly on the bipartite setup. However, as with quantum entanglement [8, 9], correlations between measurement outcomes can exhibit a much richer structure in the multipartite setup. Consider a multipartite Bell-type experiment with the $i$-th party's choice of measurement setting (input) denoted by $x_{i}=0,1$ and the corresponding outcome (output) by $a_{i}= \pm 1$. Already in the tripartite setting [10], quantum mechanics allow for correlations - a collection of joint conditional probability distributions $\vec{P}=\{P(\vec{a} \mid \vec{x})\}=\left\{P\left(a_{1} a_{2} a_{3} \mid x_{1} x_{2} x_{3}\right)\right\}$ - that cannot be reproduced even when a subset of the parties are allowed to share some nonlocal resource $\mathcal{R}$ [1, 12] 1 Such genuinely tripartite nonlocal correlations are, by definition, those that cannot be written in

\footnotetext{
1 Throughout, we focus on nonlocal resources $\mathcal{R}$ that respect the non-signaling conditions [13, 14] which dictate, e.g., that each marginal of distribution of $P_{i}^{\mathcal{R}}\left(a_{j} a_{k} \mid x_{j} x_{k}\right)$ can be defined independent of the input of the other party.
}

the so-called biseparable form:

$$
\begin{aligned}
P(\vec{a} \mid \vec{x}) & \neq \sum_{\nu} p_{\nu} P_{\nu}\left(a_{1} \mid x_{1}\right) P_{\nu}^{\mathcal{R}}\left(a_{2} a_{3} \mid x_{2} x_{3}\right) \\
& +\sum_{\mu} p_{\mu} P_{\mu}\left(a_{2} \mid x_{2}\right) P_{\mu}^{\mathcal{R}}\left(a_{1} a_{3} \mid x_{1} x_{3}\right) \\
& +\sum_{\lambda} p_{\lambda} P_{\lambda}\left(a_{3} \mid x_{3}\right) P_{\lambda}^{\mathcal{R}}\left(a_{1} a_{2} \mid x_{1} x_{2}\right)
\end{aligned}
$$

where $\sum_{i \in\{\lambda, \mu, \nu\}} p_{i}=1, p_{i} \geq 0$ for all $i \in\{\lambda, \mu, \nu\}$ and $P_{i}^{\mathcal{R}}\left(a_{j} a_{k} \mid x_{j} x_{k}\right)$ is any 2-partite correlation allowed by the resource $\mathcal{R}[11,12]$. In a Bell-type experiment, the presence of genuine multipartite nonlocality [15 19$]$ is a manifestation of genuine multipartite entanglement [8], thus facilitates the detection of the latter in a deviceindependent manner, i.e., without relying on any assumption about the measurements being performed nor the dimension of the underlying Hilbert space 2 In contrast, correlations that are biseparable, cf. Eq. (1), receive almost no attention. Apart from being a tool in the derivation of Bell-type inequalities for genuine multipartite nonlocality, is this kind of correlations interesting in its own right? Here, we answer this question affirmatively via the phenomenon of anonymous nonlocality (ANL), an intriguing feature that is only present in biseparable correlations. We will also provide evidence showing that ANL can be a powerful resource, allowing one to design device-independent quantum cryptographic protocols that can guard against a particular kind of attack by any post-quantum, but non-signaling adversary.

Biseparable correlations and anonymous nonlocality.To appreciate the peculiarity manifested by ANL, let us start by considering the simplest, tripartite scenario. Clearly, among the subsets of correlations that can be

\footnotetext{
2 It is also possible to detect genuine multipartite entanglement in a device-independent manner without the detection of genuine multipartite nonlocality. See [18, 20].
} 
decomposed in the form of the right-hand-side of Eq. (1) are those that satisfy:

$$
\begin{aligned}
P(\vec{a} \mid \vec{x}) & =\sum_{\nu} p_{\nu} P_{\nu}\left(a_{1} \mid x_{1}\right) P_{\nu}^{\mathcal{R}}\left(a_{2} a_{3} \mid x_{2} x_{3}\right), \\
& =\sum_{\mu} p_{\mu} P_{\mu}\left(a_{2} \mid x_{2}\right) P_{\mu}^{\mathcal{R}}\left(a_{1} a_{3} \mid x_{1} x_{3}\right), \\
& =\sum_{\lambda} p_{\lambda} P_{\lambda}\left(a_{3} \mid x_{3}\right) P_{\lambda}^{\mathcal{R}}\left(a_{1} a_{2} \mid x_{1} x_{2}\right),
\end{aligned}
$$

where $p_{\nu}, p_{\mu}, p_{\lambda} \geq 0$ for all $\nu, \mu$ and $\lambda$, but in contrast with Eq. (11), we now have $\sum_{\nu} p_{\nu}=\sum_{\mu} p_{\mu}=\sum_{\lambda} p_{\lambda}=1$. Eqs. (2a)-(2c) imply that the correlation can be produced without having any nonlocal collaboration between the isolated party and the remaining two parties (as a group). Naively, one may thus expect that all correlations satisfying these equations must also be Bell-local (henceforth abbreviated as local). However, there exist 21] quantum correlations that satisfy Eqs. (2a)-(2C) as well as:

$$
P(\vec{a} \mid \vec{x}) \neq \sum_{\theta} p_{\theta} P_{\theta}\left(a_{1} \mid x_{1}\right) P_{\theta}\left(a_{2} \mid x_{2}\right) P_{\theta}\left(a_{3} \mid x_{3}\right),
$$

for any conditional distributions $P_{\theta}\left(a_{i} \mid x_{i}\right)$ and any normalized weights $p_{\theta}$. In other words, $\vec{P}$ satisfying Eq. (2) is nonlocal but this nonlocality is (i) not genuinely tripartite (it is biseparable) (ii) not attributable to any of the two-partite marginals [Eqs. (2a)-(2C) imply that all marginals are local] and (iii) not attributable to any bipartition of the three parties. The nonlocality present in any correlations satisfying Eq. (2) is thus in some sense nowhere to be found!

We now provide a very simple example of correlation satisfying Eq. (2), and more generally the property of being (1) nonlocal and (2) biseparable with respect to all bipartitions in an arbitrary $n$-partite scenario. Consider the $n$-partite Greenberger-Horne-Zeilinger (GHZ) state 22] $\left|\mathrm{GHZ}_{\mathrm{n}}\right\rangle=\frac{1}{\sqrt{2}}\left(|0\rangle^{\otimes \mathrm{n}}+|1\rangle^{\otimes \mathrm{n}}\right)$ and the local measurement of $\sigma_{x}$ and $\sigma_{y}$. The resulting correlation is

$$
P(\vec{a} \mid \vec{x})=P_{\mathrm{GHZ}}^{n}(\vec{a} \mid \vec{x})=\frac{1}{2^{n}}\left[1+\cos \left(\mathrm{x} \frac{\pi}{2}\right) \prod_{i=1}^{n} a_{i}\right],
$$

where $\mathbf{x}=\sum_{i} x_{i}$, and we have identified $x_{i}=0(1)$ as the $\sigma_{x}\left(\sigma_{y}\right)$ measurement (see, eg. Eq. (23) of [23]). In Appendix A, we show that for all $n \geq 3$, n-partite correlations of the form of Eq. (3) admit a biseparable decomposition with respect to any partitioning of the $n$ parties into two groups. Specifically for $n=3$, this decomposition, cf. Eq. (2a), involves $p_{\nu}=\frac{1}{4}$ for all $\nu, P_{\nu}\left(a_{1} \mid x_{1}\right)=0,1$ and $P_{\nu}^{\mathcal{R}}\left(a_{2} a_{3} \mid x_{2} x_{3}\right)$ is the correlation associated with the so-called Popescu-Rohrlich (PR) box [13] - a hypothetical, stronger-than-quantum, but non-signaling resource 3 To see that these correlations are nonlocal, it suffices to note that Eq. (3) violates the

\footnotetext{
${ }^{3}$ In the tripartite scenario, the biseparability of the GHZ correlation was also discovered independently in 24] (see also [25]).
}

Mermin-Bell inequality [26, 27] (even maximally [28] for all odd $n \geq 3$ ). See Appendix B.

Consider now an alternative way to understand the nonlocality associated with Eq. (2). Operationally, Eq. (2C) implies that $\vec{P}$ can be produced by, e.g., party 1 signaling classically to party 2 , and all parties responding according to the information that they received and some predefined strategy $\lambda$. By symmetry of Eqs. (2a (2c), the same can be achieved by having only nonlocal collaboration between any two out of the three parties. Thus, while the correlation can be produced by having only a definite subset of parties collaborating nonlocally, the identity of these nonlocally collaborating parties is anonymous to an outsider who only has access to $\vec{P}$. Indeed, even if an outsider is given the promise that a fixed subset of the parties have collaborated nonlocally, it is impossible for him to tell if, say, party 1 and 2 have collaborated nonlocally in generating $\vec{P}$. Importantly, the anonymity present in these correlations differs from the case where a classical mixture of the different bipartitions is necessary, cf. Fig. 1 (see [29, 30] for examples of such classical anonymity). In this latter case, it is indeed possible to identify the parties that must have collaborated nonlocally, even though this identification is generally not possible at any single run of the experiment.

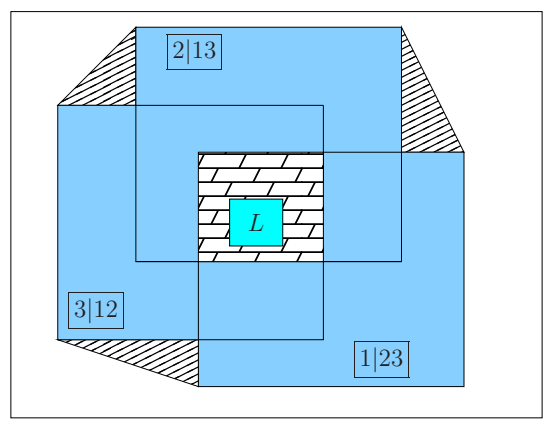

FIG. 1. (Color online) Schematic representation of the various sets of tripartite correlations. Correlations biseparable with respect to party $i$ in one group and parties $j$ and $k$ in the other lie in the (light blue) rectangle labeled by " $i \mid j k$ ". The convex hull of the three biseparable sets " $i j k$ " where $i, j, k \in\{1,2,3\}$ is represented by the filled convex region and gives correlations decomposable as the right-hand-side of Eq. (1). The blank region between the outermost box and the filled convex region represents correlations that are genuinely tripartite nonlocal. Intersection of the three biseparable subsets " $i \mid j k$ " gives correlations satisfying Eqs. (2a)-(2c); its subset featuring ANL is the tiled region while local correlations lie in the (cyan) rectangle $L$. Hatched regions represent biseparable correlations where classical mixture of different bipartitions is necessary for their production.

As remarked above, for all $n \geq 3$, the GHZ correlations of Eq. (3) are nonlocal but can nevertheless be produced by splitting the parties into any two groups, and disallowing any nonlocal collaboration between these groups. Thus, the anonymity present in these correlations is even more striking in the $n>3$ scenarios: not only are the groups of parties sharing $\mathcal{R}$ unidentifiable in an unambiguous manner, even the size of the groups are also not 
identifiable (see Fig. 2). For example, when $n=4$, the correlation satisfy:

$$
\begin{aligned}
P(\vec{a} \mid \vec{x}) & =\sum_{\lambda_{1}} q_{\lambda_{1}} P_{\lambda_{1}}\left(a_{1} \mid x_{1}\right) P_{\lambda_{1}}^{\mathcal{R}}\left(a_{2} a_{3} a_{4} \mid x_{2} x_{3} x_{4}\right), \\
& =\sum_{\lambda_{2}} q_{\lambda_{2}} P_{\lambda_{2}}\left(a_{2} \mid x_{2}\right) P_{\lambda_{2}}^{\mathcal{R}}\left(a_{1} a_{3} a_{4} \mid x_{1} x_{3} x_{4}\right), \\
& =\cdots \\
& =\sum_{\mu_{3}} q_{\mu_{3}} P_{\mu_{3}}^{\mathcal{R}}\left(a_{1} a_{4} \mid x_{1} x_{4}\right) P_{\mu_{3}}^{\mathcal{R}}\left(a_{2} a_{3} \mid x_{2} x_{3}\right), \\
P(\vec{a} \mid \vec{x}) & \neq \sum_{\theta} q_{\theta} \prod_{i=1}^{4} P_{\theta}\left(a_{i} \mid x_{i}\right),
\end{aligned}
$$

where $\sum_{\lambda_{i}} p_{\lambda_{i}}=\sum_{\mu_{j}} p_{\mu_{j}}=1, p_{i} \geq 0$ for all $i, j$, and "..." indicates other possible biseparable decompositions that have been omitted. From Eq. (4), we see that the 4-partite GHZ correlation could have been produced by having any three parties collaborating nonlocally, or any two groups of two parties collaborating nonlocally within each group. From the correlation itself, it is simply impossible to distinguish these possibilities apart (Fig. 2).

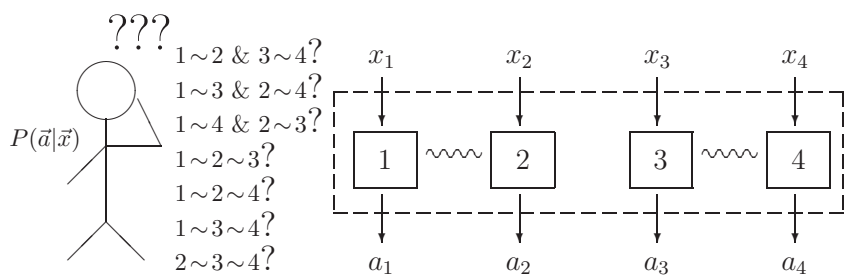

FIG. 2. ANL in the 4-partite scenario. Each participating party is abstractly represented by a box labeled by the party number. The correlations were produced by having parties $1 \& 2$, as well as $3 \& 4$ collaborated nonlocally (symbolized by " $\sim$ "). To an outsider who only has access to $\vec{a}$ and $\vec{x}$, even if one is given the promise that the correlations were produced by the four parties separated into two fixed groups, it is impossible to tell which actual partitioning of the parties generated these correlations.

Let us now briefly comment on the relationship between ANL and multipartite entanglement. Clearly, one expects that there must also be features analogous to ANL in the studies of multipartite entanglement. Indeed, the first of such examples dates back to the three-qubit bound entangled [31. SHIFT state 32] where its entanglement was dubbed delocalized 33] since it is separable with respect to all bipartitions, yet not fully separable. A more recent example 21] involves a three-qubit bound entangled state which even violates a Bell inequality, thus giving also an example of anonymous quantum correlation. An important difference between their example and the tripartite case of our GHZ example is that their correlation can be produced by a biseparable tripartite entangled state whereas ours necessarily requires a genuinely tripartite entangled state. More generally, for all odd $n \geq 3$, we show in Appendix $\mathrm{C}$ that the correlations of Eq. (3) can only be produced by genuinely $n$-partite entangled state. Our examples thus show that the generation of ANL does not require delocalized entanglement.
Perfect correlations with uniform marginals.- From Eq. (3), we see that whenever an odd number of parties measure in the $\sigma_{y}$ basis, the product of outcomes $\prod_{i} a_{i}$ gives \pm 1 with equal probability, otherwise it is either perfectly correlated or perfectly anti-correlated. Moreover, it follows from Eq. (3) that all marginal distributions of these correlations are uniformly random. Next, we present two quantum cryptography protocols that exploit these strong but anonymous correlations.

Application I: multipartite secret sharing (MSS).Imagine that $n$ parties wanted to share a secret message between any two complementary subgroups as they desire, i.e., between any subgroup of $k$ parties $(k \leq n-1)$ and the subgroup formed by the remaining parties. Suppose moreover that the shared secret is to be recovered by these subgroups only when all parties within each group collaborate (so that it is unnecessary to trust all parties within each group). A possibility to achieve this consists in: (i) the $n$ parties share (many copies of) $\left|\mathrm{GHZ}_{\mathrm{n}}\right\rangle$, (ii) each party randomly measures either the $\sigma_{x}$ or the $\sigma_{y}$ observable, (iii) the $n$ parties are randomly separated into two groups and all parties assigned to the same group collaborate to compare their inputs and outputs, (iv) both groups announce their sum of inputs, (v) parties in the same group compute the product of their measurement outcome and deduce, using Eq. (3), the shared secret bit upon learning the sum of inputs of the other group, (vi) parties in one group use the shared secret keys to encrypt the message and send it to the other.

In the device-independent setting, security analysis is carried out by treating each physical subsystem together with their measurement device as a black box; conclusions are drawn directly from the measurement statistics. Indeed, the above protocol does not rely on the assumption of a GHZ state nor the particular measurements being performed, but rather the strong correlation present in Eq. (3) - for the right combination of inputs, the product of outputs are perfectly (anti) correlated 4 Thus, the protocol essentially works by first distributing the correlated data needed to establish the secret keys, and performing the secret sharing [34] between any two complementary subgroups of the $n$ participating parties as they deem fit. Since the product of outcomes for each group is uniformly random, the protocol is secure against cheating by any dishonest parties within the group; no one can retrieve the shared key without collaborating with everyone else within the same group. What about eavesdropping by an external, post-quantum but non-signaling adversary Eve?

Since the GHZ correlations of Eq. (3) are biseparable, a naive attack by Eve may consist in preparing for the $n$ parties the biseparable, non-signaling boxes that reproduces exactly Eq. (3). For instance, in the tripartite case, in accordance with the biseparable decomposition, she would prepare with equal probability 4 different versions of a deterministic box for one of the parties, and

\footnotetext{
4 This happens in half the cases. In the other cases, the correlation is useless for key generation.
} 
correspondingly 4 different versions of a PR box for the remaining two parties. If the decomposition that she chooses matches exactly the way the parties are separated into two groups, then after step (iv), she learns exactly the key and hence the message shared by these parties However, as the grouping is decided only after the measurement phase, she can guess the bipartition correctly only with a chance of $\frac{1}{3}$ in the tripartite case, and more generally $\left(2^{n-1}-1\right)^{-1}$ in the $n$-partite scenario. Evidently, this guessing probability rapidly approaches 0 as $n$ increases, making it extremely difficult for Eve to succeed with this eavesdropping strategy for large $n$.

Application II: bipartite leakage-resilient QKD.-Next, let us describe a quantum key distribution (QKD) protocol between two parties, A and B, which is as leakageresilient 36] as one could hope for. The protocol consists of: (i) preparation of many copies of $\left|\mathrm{GHZ}_{\mathrm{n}}\right\rangle$, (ii) for each of these $n$-partite systems, a randomly chosen subset, say, $k$ of the $n$ subsystems are distributed to A, while the remaining $n-k$ subsystems are distributed to $\mathrm{B}$, (iii) for each of these subsystems, A and B randomly measure $\sigma_{x}$ or $\sigma_{y}$, (iv) both parties announce their sum of inputs, (v) for each $n$-partite system distributed from the source, A and $\mathrm{B}$ compute the product of their local measurement outcomes and deduce, using Eq. (3), the shared secret bit upon learning the sum of inputs of the other party.

As with the MSS protocol described above, the secret key is established through the perfect (anti) correlation present in the product of the outputs. Moreover, the gist of the protocol only relies on the correlation given by Eq. (3), rather than the actual state and measurement giving rise to this correlation, rendering the protocol ideal for device-independent analysis. However, in contrast with usual device-independent cryptography where leakage of information is not allowed, the above protocol is as leakage-resilient as one can hope for — the adversary Eve can certainly recover the secret key if all the output bits from either party leak to her, but if she misses merely one output bit from each party, the additional information that she gains from the leakage cannot improve her guess of the secret key. Now, if we assume that Eve has no control over how the subsystems are distributed in step (ii) 6 but otherwise only constrained by the nonsignaling principle, then as with the MSS protocol, for $n$ sufficiently large, her advantage of preparing some biseparable, non-signaling boxes for A \& B is minimal.

Discussion.- Let us now comment on some possible directions for future research. Clearly, we have only provided intuitions on why the protocols proposed above may be secure even in a device-independent setting. For odd $n \geq 3$, since the GHZ correlations violate the

\footnotetext{
${ }^{5}$ In this case, the product of outcomes for each group is a deterministic function (of the sum of inputs) known to Eve. The secret sharing protocol of Hillery et al. [35] is thus insecure against this kind of attack by a non-signaling adversary.

${ }^{6}$ Instead of this assumption, A \& B can employ additional measurement settings, cf. [5], to certify that the overall correlations indeed exhibit genuine multipartite nonlocality and they are then again not susceptible to such an attack.
}

Mermin-Bell inequality maximally (see Appendix $B$ ), the result of Franz et al. [37] implies that these correlations are necessarily monogamous with respect to any potential quantum eavesdropper. This strongly suggests that if we assume an independent-and-identically-distributed (i.i.d) scenario, a formal security proof of these protocols against a quantum adversary may be given even in the case with noisy correlations 7 and in a deviceindependent setting. Evidently, a security proof without this assumption is even more desirable, and a possible path towards this is to prove that the protocols are even secure against an adversary that is only constrained by the non-signaling principle [13]. Our arguments on why the protocols are not immediately susceptible to a straightforward attack by such an eavesdropper, despite the fact that the correlations are biseparable, is an evidence pointing in this direction.

For leakage-resilient QKD, one could also imagine, instead of the above protocol, doing an existing QKD protocol many times in parallel and then using the XOR of the secret key bits to generate the final secret key. Although such a protocol requires many more qubits to establish the final secret key, it can clearly offer high level of leakage resilience. How would such a protocol perform compared with the above protocol based on $\left|\mathrm{GHZ}_{\mathrm{n}}\right\rangle$ ? This certainly deserves some further investigation.

Coming back to ANL itself, let us note that the requirement of (1) nonlocality and (2) biseparability with respect to all bipartitions may arguably not, by themselves, imply that an outsider cannot attribute unambiguously the nonlocality to any definite subset(s) of the $n$ parties. For instance, one may start with the tripartite GHZ correlation $P_{\mathrm{GHZ}}^{3}(\vec{a} \mid \vec{x})$, cf. Eq. (3) , and trivially construct an example $P^{\prime}=P_{\mathrm{GHZ}}^{3}(\vec{a} \mid \vec{x}) \prod_{i=4}^{n} P\left(a_{i} \mid x_{i}\right)$ for arbitrary $n$ parties by introducing parties that are uncorrelated with the first three. While such an $n$-partite correlation $P^{\prime}$ indeed satisfies the two requirements stated above, one can unambiguously attribute the nonlocality present only to the three parties that give rise to $P_{\mathrm{GHZ}}^{3}(\vec{a} \mid \vec{x})$. Note, however, that such an identification is incomplete since the production of such a biseparable correlation only requires the nonlocal collaboration between two parties, and it is still impossible for an outsider to determine which two parties have collaborated nonlocally in producing the given correlation (Fig. 11 and Fig. 2). A more precise definition of ANL may thus require also a specification of the extent (size) of the nonlocal resource needed in producing the given correlation, a task that shall be pursued elsewhere [30]. For our GHZ examples, except for the cases where $n$ is even with $\frac{n}{2}$ odd, it can be shown (see Appendix D) using the result of Ref. [16] that the correlations of Eq. (3) are not triseparable, i.e., not producible by a partitioning of the parties into three groups (where only parties within the same group are allowed to collaborate nonlocally). Hence, the generation

\footnotetext{
7 Due to the noise robustness of the Mermin-Bell violation of $\vec{P}_{\mathrm{GHZ}}^{n}(\vec{a} \mid \vec{x})$, the ANL of $\vec{P}_{\mathrm{GHZ}}^{n}(\vec{a} \mid \vec{x})$ is also extremely robust to noise.
} 
of these correlations indeed requires the nonlocal collaboration of at least $\left\lceil\frac{n}{2}\right\rceil$ parties in one group; an analogous statement for the remaining cases would be desirable.

\section{ACKNOWLEDGMENTS}

We are grateful to David Jennings for suggesting the terminology "Anonymous Nonlocality", and to Rotem Arnon-Friedman, Jean-Daniel Bancal, Nicolas Brunner, Stefano Pironio as well as Renato Renner for stimulating discussions. This work is supported by the Swiss NCCR "Quantum Science and Technology", and the CHISTERA DIQIP. FJC acknowledges support from the John Templeton Foundation. YCL and FJC contribute equally towards this work.

\section{Appendix A: An explicit biseparable decomposition of the $n$-partite GHZ correlations}

For the $n$-partite GHZ state and the situation where all parties measure either the 0th-observable $\sigma_{x}$ or the 1st observable $\sigma_{y}$, the resulting correlation of Eq. (3) can be rewritten in terms of the correlator, i.e., the expectation value of the product of outcomes 8

$$
E(\vec{x})=\sum_{a_{1}^{\prime}, a_{2}^{\prime}, \ldots, a_{n}^{\prime}=0,1}(-1)^{\sum_{i} a_{i}^{\prime}} P\left(\vec{a}^{\prime} \mid \vec{x}\right)=\cos \left(\mathrm{x} \frac{\pi}{2}\right)
$$

where for conciseness of subsequent presentation we have used, instead, $a_{i}^{\prime}=\frac{a_{i}+1}{2}=0,1$ to denote the output and as before, $\mathbf{x}=\sum_{i} x_{i}$ to denote the sum of inputs. Note that all the full $n$-partite correlators depend only on the parity of $\mathbf{x}$ and $\mathbf{x} / 2$ whereas all the marginal correlators vanish.

Here we give a proof that the above correlation is biseparable with respect to all bipartitions whenever parties in each group are allowed to share arbitrary post-quantum but non-signaling (NS) resources, while parties in different groups can only be correlated through shared randomness. Note that the biseparability of Eq. (3) under the NS constraint implies that if parties in the same group are allowed to share a stronger resource, such as a Svetlichny resource [10], or some other one-way signaling resource discussed in Refs. 11, 12], the correlation must remain biseparable.

Let us define the four families of $n$-partite NS boxes, labeled by $\mu_{1}, \mu_{2}, \mu_{3}$ and $\mu_{4}$ :

$$
\begin{aligned}
P_{\mu_{1}}^{n}\left(\vec{a}^{\prime} \mid \vec{x}\right) & =\frac{1}{2^{n-1}} \delta_{\sum_{i=1}^{n} a_{i}^{\prime}-H_{0}^{n}(\vec{x})-H_{3}^{n}(\vec{x}) \bmod 2,} \\
P_{\mu_{2}}^{n}\left(\vec{a}^{\prime} \mid \vec{x}\right) & =\frac{1}{2^{n-1}} \delta_{\sum_{i=1}^{n} a_{i}^{\prime}-H_{0}^{n}(\vec{x})-H_{1}^{n}(\vec{x}) \bmod 2,} \\
P_{\mu_{3}}^{n}\left(\vec{a}^{\prime} \mid \vec{x}\right) & =\frac{1}{2^{n-1}} \delta_{\sum_{i=1}^{n} a_{i}^{\prime}-H_{1}^{n}(\vec{x})-H_{2}^{n}(\vec{x}) \bmod 2}, \\
P_{\mu_{4}}^{n}\left(\vec{a}^{\prime} \mid \vec{x}\right) & =\frac{1}{2^{n-1}} \delta_{\sum_{i=1}^{n} a_{i}^{\prime}-H_{2}^{n}(\vec{x})-H_{3}^{n}(\vec{x}) \bmod 2},
\end{aligned}
$$

\footnotetext{
8 To arrive at this $n$-partite correlator, see, eg., Eq. (23) of [23].
}

where $H_{\ell}^{n}(\vec{x})=\sum_{j=0}^{\left\lfloor\frac{n-\ell}{4}\right\rfloor} F(4 j+\ell, \vec{x})$,

$$
F(k, \vec{x})=\sum_{G} \prod_{i \in G} x_{i} \prod_{j \in G^{\prime}}\left(x_{j}+1\right)
$$

and the sum $\sum_{G}$ is over all $G \subseteq[n]=\{1,2, \ldots, n\}$ with group size $|G|=k$, and $G^{\prime}$ is the complement of $G$ in $[n]$. Essentially, each term involved in the summand in $F(k, \vec{x})$, and hence $H_{\ell}^{n}(\vec{x})$ defines a distinct combination of inputs $\vec{x}=\vec{x}^{\prime}$ such that $H_{\ell}^{n}\left(\vec{x}^{\prime}\right)=1 \bmod 2$, and hence making the outputs anti-correlated. For instance, $F(0, \vec{x})$ only makes a nontrivial combination to $H_{0}^{n}(\vec{x})$ if all the inputs $x_{i}$ are 0 .

From Eq. (A1), it is easy to verify that for all $1 \leq$ $k \leq n-1$, the $k$-partite marginals of $P_{j}^{n}\left(\vec{a}^{\prime} \mid \vec{x}\right)$ are $1 / 2^{k}$ and these correlations indeed define NS probability distributions. Moreover, from Eq. (A1) and these marginal distributions, one can show that these NS boxes give rise to vanishing marginal correlators and the following full $n$-partite correlators:

$$
\begin{aligned}
& E(\vec{x})_{\mu_{1}}=(-1)^{H_{0}^{n}(\vec{x}) \oplus H_{3}^{n}(\vec{x})}=-E(\vec{x})_{\mu_{3}}, \\
& E(\vec{x})_{\mu_{2}}=(-1)^{H_{0}^{n}(\vec{x}) \oplus H_{1}^{n}(\vec{x})}=-E(\vec{x})_{\mu_{4}},
\end{aligned}
$$

where in Eq. A3), $\oplus$ denotes sum modulo 2 and in arriving at the second equality in each line, we have employed the identity $\sum_{j=0}^{n} F(j)=1$ that holds for all

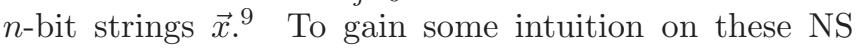
boxes, we note that for $n=1$, the $\mu_{1 / 3}$ boxes correspond to the deterministic strategies $a^{\prime}=x \oplus 1$ and $a^{\prime}=x$ whereas the $\mu_{2 / 4}$ boxes correspond to the deterministic strategies $a^{\prime}=1$ and $a^{\prime}=0$. Similarly, for $n=2$, the $\mu_{1 / 3}$ boxes correspond to the PR boxes defined by $a_{1}^{\prime}+a_{2}^{\prime}=\left(x_{1}+1\right)\left(x_{2}+1\right)$ and $a_{1}^{\prime}+a_{2}^{\prime}=\left(x_{1}+1\right)\left(x_{2}+1\right) \oplus 1$ whereas the $\mu_{2 / 4}$ boxes correspond to the PR boxes defined by $a_{1}^{\prime}+a_{2}^{\prime}=x_{1} x_{2} \oplus 1$ and $a_{1}^{\prime}+a_{2}^{\prime}=x_{1} x_{2}$. For $n=3$, all these NS boxes correspond to some version of NS box 46 described in Ref. 24]. It is conceivable that these boxes are extremal NS distributions for all $n$.

To reproduce the correlations given in Eq. (3) using biseparable $\mathcal{N S}$ resources with $k$ parties in one group and the remaining $(n-k)$ parties in the other group, it suffices to consider an equal-weight mixture of the following four strategies:

1. The group of $k$ parties share the $k$-partite version of the $\mu_{1}$ box and the remaining parties share the $(n-k)$-partite version of the $\mu_{2}$ box.

2. The group of $k$ parties share the $k$-partite version of the $\mu_{3}$ box and the remaining parties share the $(n-k)$-partite version of the $\mu_{4}$ box.

3. The group of $k$ parties share the $k$-partite version of the $\mu_{2}$ box and the remaining parties share the $(n-k)$-partite version of the $\mu_{1}$ box.

\footnotetext{
9 This last sum involves all possible combinations of inputs and thus for all input bit strings $\vec{x}$, there is exactly one term in the expression that does not vanish, therefore giving the identity.
} 
4. The group of $k$ parties share the $k$-partite version of the $\mu_{4}$ box and the remaining parties share the $(n-k)$-partite version of the $\mu_{3}$ box.

For $n=3$, the above strategy corresponds to a mixture of 4 different versions of the NS box 2 in Ref. [24]. In general, to verify that the above strategy indeed gives rise to Eq. (3), we first remark that each of these strategies also reproduces Eq. (3) for the case when $\sum_{i} x_{i}$ is even. To see this, we use the fact that NS box $\mu_{1}$ gives anti-correlation (i.e., expectation value -1) only if either $\sum_{i} x_{i} / 2$ or $\left(1+\sum_{i} x_{i}\right) / 2$ is even; NS box $\mu_{2}$ gives anticorrelation only if $\sum_{i} x_{i} / 2$ is even or $\left(1+\sum_{i} x_{i}\right) / 2$ is odd; NS box $\mu_{3}$ gives anti-correlation only if either $\sum_{i} x_{i} / 2$ or $\left(1+\sum_{i} x_{i}\right) / 2$ is odd; NS box $\mu_{4}$ gives anti-correlation only if $\sum_{i} x_{i} / 2$ is odd or $\left(1+\sum_{i} x_{i}\right) / 2$ is even. Moreover, since strategy 1 and 3 are such that the correlation produced by parties in the same group are exactly opposite (likewise for strategy 2 and 4 ), we see that all the less-than- $n$-partite correlators, as well as the full $n$ partite correlator when $\sum_{i=1}^{n} x_{i}$ is odd, indeed vanishes as claimed.

\section{Appendix B: Mermin-Bell violation of the GHZ correlations}

Here, we compute the quantum expectation value of the GHZ correlations for the Mermin Bell inequality [26, 27] (here written in the form derived in [23] 10

$$
\left|\mathcal{B}_{ \pm}^{n}\right|=2^{\frac{1-n}{2}}\left|\sum_{\vec{x} \in\{0,1\}^{n}} \cos \left\{\frac{\pi}{4}[1 \pm(n-2 \mathbf{x})]\right\} E(\vec{x})\right| \leq 1 .
$$

The above Bell expression can be rewritten as:

$$
\begin{aligned}
2^{\frac{n-1}{2}}\left|\mathcal{B}_{ \pm}^{n}\right|= & \left|\sum_{\vec{x} \in\{0,1\}^{n}} \cos \left\{\frac{\pi}{4}[1 \pm(n-2 \mathbf{x})]\right\} E(\vec{x})\right|, \\
= & \mid \sum_{\vec{x} \in\{0,1\}^{n}} \cos \left[\frac{\pi}{4}(1 \pm n)\right] \cos \left(\mathbf{x} \frac{\pi}{2}\right) E(\vec{x}) \\
& \pm \sum_{\vec{x} \in\{0,1\}^{n}} \sin \left[\frac{\pi}{4}(1 \pm n)\right] \sin \left(\mathbf{x} \frac{\pi}{2}\right) E(\vec{x}) \mid .
\end{aligned}
$$

For the GHZ correlation of Eq. (3), this simplifies to

$$
\begin{aligned}
\left|\mathcal{B}_{ \pm}^{n}\right| & =2^{\frac{1-n}{2}}\left|\sum_{\vec{x} \in\{0,1\}^{n}, \mathbf{x} \text { even }} \cos \left[\frac{\pi}{4}(1 \pm n)\right] \cos ^{2}\left(\mathbf{x} \frac{\pi}{2}\right)\right|, \\
& =2^{\frac{n-1}{2}}\left|\cos \left[\frac{\pi}{4}(1 \pm n)\right]\right|
\end{aligned}
$$

giving

$$
\max _{ \pm}\left|\mathcal{B}_{ \pm}^{n}\right|=\left\{\begin{array}{r}
2^{\frac{n-1}{2}}: n \text { odd } \\
2^{\frac{n-2}{2}}: n \text { even }
\end{array}\right.
$$

i.e., achieving maximal [28] possible quantum value of $\left|\mathcal{B}_{ \pm}^{n}\right|$ for odd $n$.

$10 \mathcal{B}_{+}^{n}$ is the same Bell expression as the usual one obtained through the recursive formula [27]; it can also be obtained by flipping all the inputs in $\mathcal{B}_{-}^{n}$.

\section{Appendix C: Quantum biseparable bound of the $n$-partite Mermin-Bell expression}

For arbitrary odd $n \geq 3$, the Mermin-Bell expression $\mathcal{B}_{+}^{n}$ given on the left-hand-side of Eq. (B1) is equivalent to a special case of a general family of permutationally invariant Bell expression described in Eq. (22) of [18],

$$
\Omega_{n, 2,2 ; \delta_{\mathbf{x}, 0} \cdot r}=2^{n-2}-2^{\frac{n-3}{2}} \mathcal{B}_{+}^{n}
$$

From Eq. (23) of Ref. [18], it can be shown that the above expression admits the following upper bound on the quantum biseparable bound:

$$
\Omega_{n, 2,2 ; \delta_{\mathbf{x}, 0} \cdot r} \geq 2^{n-3}(2-\sqrt{2}) .
$$

Combining these two equations and after some straightforward computations, we get the following upper bound on the quantum biseparable bound for the Mermin-Bell expression:

$$
\mathcal{B}_{+}^{n} \leq 2^{\frac{n}{2}-1}
$$

For arbitrary even $n \geq 2$, the Mermin-Bell expression $\mathcal{B}_{+}^{n}$ given on the left-hand-side of Eq. (B1) is equivalent to the following Bell expression described in Eq. (1) of Ref. [18],

$$
\mathcal{I}_{n, 2,2}=2^{n-1}-2^{\frac{n-2}{2}} \mathcal{B}_{+}^{n}
$$

From Eq. (25) of Ref. [18], we know that the above expression admits the following upper bound on the quantum biseparable bound:

$$
\mathcal{I}_{n, 2,2} \geq 2^{n-2} .
$$

Combining these two equations, we arrive, again, at Eq. (C3).

To see that the biseparable bound of Eq. (C3) is tight, it suffices to note that the biseparable quantum state

$$
|\psi\rangle=\left|\mathrm{GHZ}_{n-1}\right\rangle \otimes|0\rangle
$$

and the local observables

$$
\begin{aligned}
& A_{x_{i}}=\cos \alpha_{x_{i}} \sigma_{x}+\sin \alpha_{x_{i}} \sigma_{y} \quad \forall i=1, \ldots, n-1, \\
& A_{x_{i}}=\beta_{x_{i}} \mathbb{1} \quad \text { for } \quad i=n .
\end{aligned}
$$

with $\alpha_{0}=-\frac{\pi}{4(n-1)}, \alpha_{1}=-\frac{\pi}{2}-\frac{\pi}{4(n-1)}, \beta_{0}=-\sqrt{2} \sin \frac{n \pi}{4}$, and $\beta_{1}=\sqrt{2} \cos \frac{n \pi}{4}$ indeed give rise to a quantum value of $\mathcal{B}_{+}^{n}$ of $2^{\frac{n}{2}-1}$. Since $\mathcal{B}_{-}^{n}$ can be obtained from $\mathcal{B}_{+}^{n}$ by flipping all the inputs, the same quantum biseparable bound holds for $\mathcal{B}_{-}^{n}$.

Since the GHZ correlations of Eq. (3) give Eq. (B2), we see that for odd $n$, the generation of these correlations necessarily requires a genuinely $n$-partite entangled state, independent of the underlying Hilbert space dimension. 


\section{Appendix D: $m$-separability and multipartite nonlocality underlying the $n$-partite GHZ correlations}

For odd $n$, we know from the main theorem of [16] that a quantum violation of $\left|\mathcal{B}_{ \pm}^{n}\right|=2^{\frac{n-1}{2}}$ implies that it is impossible to reproduce these GHZ correlations using any 3-separable resource (i.e., a partitioning of the parties into three groups, and where the parties within each group can share even arbitrary nonlocal resource).

For even $n$, let us evaluate the the quantum value of the following Bell expression [16]:

$$
\begin{aligned}
\left|\mathcal{B}_{\Sigma}^{n}\right| & =\frac{1}{\sqrt{2}}\left|\mathcal{B}_{+}^{n}+\mathcal{B}_{-}^{n}\right|, \\
& =\frac{1}{\sqrt{2}}\left|\sum_{\vec{x} \in\{0,1\}^{n}} \sum_{s=0,1} \cos \frac{\pi}{4}\left[1+(-1)^{s}(n-2 \mathbf{x})\right] E(\vec{x})\right|, \\
& =\frac{1}{\sqrt{2}}\left|\sum_{\vec{x} \in\{0,1\}^{n}} 2 \cos \frac{\pi}{4} \cos \left[\frac{\pi}{4}(n-2 \mathbf{x})\right] E(\vec{x})\right|,
\end{aligned}
$$

$$
=\left|\sum_{\vec{x} \in\{0,1\}^{n}} \cos \left[\frac{\pi}{4}(n-2 \mathbf{x})\right] E(\vec{x})\right| .
$$

For even $n$ and $E(\vec{x})$ of Eq. (3), this becomes

$$
\left|\sum_{\vec{x} \in\{0,1\}^{n}, \mathbf{x} \text { even }} \cos \frac{n \pi}{4} \cos ^{2} \mathbf{x} \frac{\pi}{2}\right|=2^{n-1}\left|\cos \frac{n \pi}{4}\right|,
$$

giving a value of $2^{n-1}$ for even $\frac{n}{2}$ and 0 for odd $\frac{n}{2}$.

Again, note from the main theorem of Ref. 16] that for even $n$, any correlation producible by a partition of the $n$ parties into 3 groups (each sharing some Svetlichny resource $\mathcal{S} 10-12$ ) can at most give a value of $\mathcal{B}_{\Sigma}^{n}=2^{n-2}$. This means that, as with odd $n$, the $n$-partite GHZ correlation for even $n$ with even $\frac{n}{2}$ is not producible by any partition of the parties into 3 groups, even if parties in each group are allowed to share whatever nonlocal resource.

Together with the biseparable decomposition obtained for these correlations, the above results on $m$-separability imply that for (1) odd $n$ and (2) even $n$ with even $\frac{n}{2}$, generation of the GHZ correlations of Eq. (3) requires the nonlocal collaboration of at least $\left\lceil\frac{n}{2}\right\rceil$ parties in one group.
[1] J. S. Bell, Physics (NY) 1 (3), 195-200 (1964).

[2] R. Cleve, P. Høyer, B. Toner, and J. Watrous, in Proceedings of the 19th IEEE Annual Conference on Computational Complexity 2004, Amherst, MA, (IEEE Conference Proceedings, New York, 2004), pp. 236-249.

[3] H. Buhrman, R. Cleve, and W. van Dam, SIAM J. Comput. 30, 1829 (2001); Č. Brukner, M. Żukowski, J.W. Pan, and A. Zeilinger, Phys. Rev. Lett. 92, 127901 (2004).

[4] A. K. Ekert, Phys. Rev. Lett. 67, 661 (1991); J. Barrett, L. Hardy, and A. Kent, Phys. Rev. Lett. 95, 010503 (2005); A. Acín, N. Gisin, and Ll. Masanes, Phys. Rev. Lett. 97, 120405 (2006); A. Acín, N. Brunner, N. Gisin, S. Massar, S. Pironio, and V. Scarani, Phys. Rev. Lett. 98, 230501 (2007).

[5] A. Acín, S. Massar, and S. Pironio, New J Phys. 8, 126 (2006).

[6] R. Colbeck, PhD Dissertation, University of Cambridge (2007), arXiv:0911.3814 (2009); S. Pironio, A. Acín, S. Massar, A. Boyer de la Giroday, D. N. Matsukevich, P. Maunz, S. Olmschenk, D. Hayes, L. Luo, T. A. Manning, and C. Monroe, Nature (London) 464, 1021 (2010).

[7] N. Brunner, D. Cavalcanti, S. Pironio, V. Scarani, and S. Wehner, Rev. Mod. Phys. 86, 419 (2014).

[8] R. Horodecki, P. Horodecki, M. Horodecki, and K. Horodecki, Rev. Mod. Phys. 81, 865 (2009); O. Gühne and G. Tóth, Phys. Rep. 474, 1 (2009).

[9] M. P. Seevinck and J. Uffink, Phys. Rev. A 78, 032101 (2008); S. Szalay and Z. Kökényesi, Phys. Rev. A 86, 032341 (2012).

[10] G. Svetlichny, Phys. Rev. D 35, 3066 (1987).

[11] R. Gallego, L. E. Würflinger, A. Acín, and M. Navascués, Phys. Rev. Lett. 109, 070401 (2012).

[12] J.-D. Bancal, J. Barrett, N. Gisin and S. Pironio, Phys. Rev. A 88, 014102 (2013).
[13] S. Popescu and D. Rohrlich, Found. Phys. 24, 379 (1994).

[14] J. Barrett, N. Linden, S. Massar, S. Pironio, S. Popescu, and D. Roberts, Phys. Rev. A 71, 022101 (2005).

[15] D. Collins, N. Gisin, S. Popescu, D. Roberts, and V. Scarani, Phys. Rev. Lett. 88, 170405 (2002); M. Seevinck and G. Svetlichny, Phys. Rev. Lett. 89, 060401 (2002); N. S. Jones, N. Linden, and S. Massar, Phys. Rev. A 71, 042329 (2005); J.-D. Bancal, N. Brunner, N. Gisin, and Y.-C. Liang, Phys. Rev. Lett. 106, 020405 (2011); J.-L. Chen, D.-L. Deng, H.-Y. Su, C. Wu, and C. H. Oh, Phys. Rev. A 83, 022316 (2011).

[16] J.-D. Bancal, C. Branciard, N. Gisin and S. Pironio, Phys. Rev. Lett. 103, 090503 (2009).

[17] L. Aolita, R. Gallego, A. Cabello, and A. Acín, Phys. Rev. Lett. 108, 100401 (2012).

[18] J.-D. Bancal, C. Branciard, N. Brunner, N. Gisin, and Y.-C. Liang, J. Phys. A: Math. Theor. 45, 125301 (2012).

[19] Q. Chen, S. Yu, C. Zhang, C. H. Lai, and C. H. Oh, Phys. Rev. Lett. 112, 140404 (2014).

[20] J.-D. Bancal, N. Gisin, Y.-C. Liang and S. Pironio, Phys. Rev. Lett. 106, 250404 (2011); K. F. Pál and T. Vértesi, Phys. Rev. A 83, 062123 (2011); T. Moroder, J.-D. Bancal, Y.-C. Liang, M. Hofmann, and O. Gühne, Phys. Rev. Lett. 111, 030501 (2013).

[21] T. Vértesi and N. Brunner, Phys. Rev. Lett. 108, 030403 (2012).

[22] D. M. Greenberger, M. A. Horne, A. Zeilinger, in "Bell's Theorem, Quantum Theory, and Conceptions of the Universe", edited by M. Kafatos (Kluwer, Dordrecht, 1989), 69-72; N. D. Mermin, Phys. Rev. Lett. 65, 3373 (1990).

[23] J. J. Wallman, Y.-C. Liang, and S. D. Bartlett, Phys. Rev. A 83, 022110 (2011).

[24] S. Pironio, J.-D. Bancal, and V. Scarani, J. Phys. A: Math. Theor. 44, 065303 (2011).

[25] J. L. Cereceda, Phys. Rev. A 66, 024102 (2002); P. 
Mitchell, S. Popescu, and D. Roberts, Phys. Rev. A 70, 060101(R) (2004).

[26] N. D. Mermin, Phys. Rev. Lett. 65, 1838 (1990); M. Ardehali, Phys. Rev. A 46, 5375 (1992).

[27] S. M. Roy and V. Singh, Phys. Rev. Lett. 67, 2761 (1991); A. V. Belinskiǐ and D. N. Klyshko, Phys. Usp. 36653 (1993); N. Gisin and H. Bechmann-Pasquinucci, Phys. Lett. A 246, 1 (1998).

[28] R. F. Werner and M. M. Wolf, Phys. Rev. A 61, 062102 (2000).

[29] F. J. Curchod, Y.-C. Liang and N. Gisin, J. Phys A: Math. Theor. (in press); arXiv:1310.7598 (2013).

[30] F. J. Curchod et al. (in preparation).

[31] M. Horodecki, P. Horodecki, and R. Hodoecki, Phys. Rev. Lett. 80, 5239 (1998).

[32] C. H. Bennett, D. P . DiVincenzo, T. Mor, P. W. Shor, J. A. Smolin, B. M. Terhal, Phys. Rev. Lett. 82, 5385
(1999)

[33] D. P. DiVincenzo, T. Mor, P. W. Shor, J. A. Smolin, and B. M. Terhal, Commun. Math. Phys. 238, 379 (2003).

[34] B. Schneier, Applied Cryptography (Wiley, New York, 1996), p. 70-74; J. Gruska, Foundations of Computing (Thomson Computer Press, London, 1997), p. 504.

[35] M. Hillery, V. Bužek, and A. Berthiaume, Phys. Rev. A 59, 1829 (1999).

[36] S. Dziembowski and K. Pietrzak, in Proceedings of IEEE 49th Annual IEEE Symposium on Foundations of Computer Science, p. 293-302; F.-X. Standaert, O. Pereira, Y. Yu, J.-J. Quisquater, M. Yung, and E. Oswald, in Towards Hardware-Intrinsic Security (Springer, Berlin, 2010), p. 99-134; F. G. Lacerda, J. M. Rennes, and R. Renner, arXiv:1404.7516v1 (2014).

[37] T. Franz, F. Furrer, and R. F. Werner, Phys. Rev. Lett. 106, 250502 (2011). 